\title{
LA INFLUENCIA DE LAS DENOMINACIONES DE ORIGEN EN EL DESARROLLO ECONÓMICO DE LA REGIÓN SURESTE: CASO TABASCO.
}

Cinthia Odet López Hernández*, Aída Armenta Ramírez*, Yazmín Isolda Álvarez García ${ }^{\star \star \star ~}$

*Licenciada en Mercadotecnia por la Universidad Interamericana para el Desarrollo. Estudiante de Maestría en Ciencias en Gestión del Desarrollo, de la Universidad Juárez Autónoma de Tabasco en la División Académica de Ciencias Económico Administrativas.

**Doctora en Economía. Profesora - Investigadora, en la División Académica de Ciencias Económico Administrativas de la Universidad Juárez Autónoma de Tabasco.

***Doctora en Estudios Jurídicos. Profesora - Investigadora en la División Académica de Ciencias de la Salud de la Universidad Juárez Autónoma de Tabasco.

Dirección para recibir correspondencia: odette201188@gmail.com 

LA REGIÓN SURESTE: CASO TABASCO.

\section{RESUMEN}

Objetivo: Conocer el impacto de la Denominación de Origen en el desarrollo económico de la Región Sureste, para lo cual se atienden los casos de las denominaciones "Cacao Grijalva" y "Chile Habanero de la Península de Yucatán" perteneciente a los Estados Tabasco con respecto al cacao y Yucatán, Campeche y Quintana Roo con respecto al Chile habanero.

Material y método: Los métodos utilizados fueron documental, observación directa y método hipotético-deductivo, de igual forma se llevó a cabo un análisis comparativo entre ambas denominaciones "Cacao Grijalva" y "Chile Habanero de la Península de Yucatán". Dentro de los instrumentos se utilizó un cuestionario cerrado aplicado a los pequeños productores de cacao pertenecientes a la subregión centro de la región Grijalva.

Resultados: Como resultado de la aplicación del cuestionario cerrado a los productores de cacao de la subregión, se detecta que éstos desconocen en un primer punto en qué consiste la figura de la Denominación de Origen, segundo que el Estado de Tabasco cuenta con el otorgamiento de ésta figura, de lo cual resulta relevante el hecho que la Denominación en cuestión no se encuentra identificada plenamente como parte del Estado por el nombre que se utilizó para su otorgamiento "Grijalva", esta denominación crea confusión en los productores, por consecuencia los productores desconocen las ventajas que con lleva el que Tabasco cuente con este reconocimiento, y por último indican que no pertenecen a ninguno de los programas de apoyo para el cultivo de este producto.

Conclusiones: El impacto de la Denominación de Origen en el desarrollo económico de la Región Sureste, tiene dos vertientes, en el caso de la solicitud por parte de los mismos productores de manera conjunta, representa una mayor estabilidad para la producción, comercialización y otorgamiento de recursos los cuales se ven reflejados en el propio estado y en los mismos productores. En el caso de la solicitud de manera unilateral, no existe el sentido de pertenencia por parte de los productores, existe falta de conocimiento, consenso y compromiso, por lo que el impacto en el estado de Tabasco es totalmente diferente en el impacto económico del estado de Yucatán, ya que la denominación de origen de cacao Grijalva no cumple con la función de la identificar el producto y la región de la cual es originaria, Grijalva hace referencia al río Grijalva en el cual se llevan a cabo actividades deportivas acuáticas, la pesca de robalo, éste río no es exclusivo del Estado de Tabasco, se incluye en su recorrido el Estado de Chiapas. 

LA REGIÓN SURESTE: CASO TABASCO.

Palabras clave: Indicación geográfica. Denominación de origen. Marca colectiva. Cacao Grijalva. Chile habanero de la Península de Yucatán.

\section{INTRODUCCIÓN}

\section{Marco jurídico de la figura de denominación de origen}

La Indicación Geográfica (IG), de acuerdo a la Organización Mundial de la Propiedad Intelectual, es un signo utilizado para productos que tienen un origen geográfico concreto y cuyas cualidades, reputación y características se deben esencialmente a su lugar de origen. Por lo general, la IG consiste en el nombre del lugar de origen de los productos. Un ejemplo típico son los productos agrícolas que poseen cualidades derivadas de su lugar de producción y están sometidos a factores geográficos específicos, como el clima y el terreno (OMPI, 2016a).

Los productos para los cuales se puede solicitar una IG son los productos agrícolas, los alimentos, los vinos y las bebidas espirituosas, la artesanía y los productos industriales.

Las principales formas de proteger una IG se encuentran las marcas colectivas o de certificación y las Denominaciones de Origen. Algunos nombres como Champagne, Cognac, Roquefort, Chianti, Porto (oporto), Tequila y Darjeeling, estos productos además se asocian con productos de determinada índole, calidad y origen geográfico (OMPI, 2016b).

De acuerdo a la Legislación de Propiedad Industrial Mexicana se entiende por DO, el nombre de una región geográfica del país que sirva para designar un producto originario de la misma, y cuya calidad o característica se deba exclusivamente al medio geográfico, comprendido en éste los factores naturales y los humanos (IMPI, 2016).

Las IG renuevan su valor, dirigiéndose principalmente al mercado de las "especialidades alimentarias", con productos diferenciados y valor agregado. Presentan también ventajas económicas, dado que facilitan la coordinación de la cadena de producción alimentaria en torno al origen geográfico y a las prácticas culturales y tecnologías locales, que determinan una calidad específica y propia; promueven las alianzas o asociaciones de las PYMES (lo que facilita la llegada a los mercados -tanto local como de exportación, al asegurar volumen y calidad homogénea-) y la exportación de distintas especialidades, generando riqueza al país y a las respectivas economías regionales. También resultan apreciables desde el 

LA REGIÓN SURESTE: CASO TABASCO.

enfoque ambiental, puesto que constituyen el nexo entre el producto y el terruño, y aseguran técnicas de producción sustentables (Jatib, 1996).

A diferencia de los otros tipos de signos distintivos regulados por la Ley de la Propiedad Industrial, como el nombre comercial, la marca o el aviso comercial, en los que una entidad jurídica específica, por lo general un prestador de servicios, industrial o comerciante, particular o privado, se constituye en titular de los derechos, en el caso de la DO tal titularidad se diluye (Ortíz, 2015).

El Estado mexicano como titular, puede autorizar el uso de la DO mediante una autorización que expida el Instituto Mexicano de la Propiedad Industria (IMPI), esta autorización tendrá una duración de diez años, contados a partir de la presentación de la solicitud de autorización de la DO y puede renovarse por periodos iguales.

La DO se orienta a proclamar la exclusividad sobre un genérico, ajustándola a los límites y condiciones que la propia ley define. En sí el contraste se perfila por la circunstancia de que, mientras una objeción natural para el registro de marcas es el que éstas consistan en expresiones genéricas, en la DO la protección se gesta, de algún modo, sobre un genérico de condiciones extraordinarias.

Estas condiciones extraordinarias son tres: que se trate del nombre de una región geográfica del país; que dicha denominación sirva para designar un producto originario de dicha región; que la calidad o características del producto obedezca al medio geográfico, comprendiéndose en ello los factores naturales y humanos.

Actualmente, México cuenta con quince DO protegidas por la legislación en materia de propiedad industrial de nuestro país, estas DO's son reconocidas en diferentes instrumentos internacionales de los que México es parte. La primera DO de México fue la denominación del Tequila en 1974, la última DO es el Cacao Grijalva en 2016 (IMPI, 2016).

Tal como lo señala, el Dr. Mauricio Jalife, cuando cierto producto de una localidad logra posicionamiento a nivel internacional, los ingresos que pueden derramarse a dicha colectividad cuando se logra que la DO en cuestión sea tutelada en esta forma, son muy cuantiosos, ya que se reserva en su favor el empleo exclusivo de la misma. Se constituye, así, una ventaja competitiva de enorme valor, que se proyecta en el tiempo para generar beneficios permanentemente a la población, región o país de que es originaria (Jalife, 2016). 

LA REGIÓN SURESTE: CASO TABASCO.

Al momento de solicitar la DO, se debe realizar una descripción detallada del producto o los productos terminados que abarca la denominación incluyendo sus características, componentes, formas de extracción y procesos de producción o elaboración. Cuando sea determinante para establecer la relación entre la denominación y el producto, se señala las normas oficiales establecidas por la Secretaría de Economía a que debe sujetarse el producto, su forma de extracción, sus procesos de elaboración o producción y sus modos de empaque, embalaje o embasamiento. De igual forma se debe señalar el lugar o los lugares de extracción, producción o elaboración del producto que se trate de proteger con la denominación de origen y la delimitación del territorio de origen, atendiendo a los caracteres geográficos y a las divisiones políticas (IMPI, 2016).

La vigencia de la declaración de protección de una DO está determinada por la subsistencia de las condiciones que motivaron y solo deja de surtir efectos por otra declaración del Instituto.

En la siguiente tabla se detallan las DO's en México y el producto que protegen.

Tabla 1.

Denominaciones de Origen Mexicanas

\begin{tabular}{llrl}
\hline $\begin{array}{l}\text { Denominación de } \\
\text { Origen }\end{array}$ & Producto & Región \\
\hline Ámbar de Chiapas & $\begin{array}{l}\text { Ámbar piedra } \\
\text { semipreciosa } \\
\text { origen vegetal. }\end{array}$ & $\begin{array}{l}12 \text { Poblaciones de Chiapas: Ostuacán, } \\
\text { de }\end{array}$ & $\begin{array}{l}\text { Tecpatán, Ocozocoautla, Pueblo Nuevo, } \\
\text { Huitiupán, San Andrés Duraznal, Pantelhó, } \\
\end{array}$ \\
& $\begin{array}{l}\text { San Lucas, Totolapa, Salto de Agua, } \\
\text { Palenque y Simojovel. }\end{array}$
\end{tabular}

Arroz del Estado de Arroz palay, integral 22 Regiones de Morelos: Cuernavaca, Morelos o moreno y pulido, Jiutepec, Yautepec, Cuautla, Temixco, subproductos de Miacatlán, Xochitepec, Coatlán del Rio, arroz: cascarilla, Tetecala, Mazatepec, Amacuzac, Puente de medio grano, tres Ixtla, Jojutla, Zacatepec, Tlaquitenango, cuartos de grano, Tepalcingo, Axochiapan, Lonacatepec, granillo, salvadillo y Jantetelco, Ciudad Ayala, Tlaltizapán y harina. Emiliano Zapata.

Bacanora Bebida Alcohólica Sonora (35 municipios) regional de Sonora.

Cacao Grijalva Cacao verde o 11 estados de Tabasco, Cárdenas, Centro, tostado/molido de la Comalcalco, Cunduacán, Huimanguillo, especie Theobroma Jalapa, Jalpa de Méndez, Nacajuca, Paraíso, Cacao. Tacotalpa y Teapa. 

LA REGIÓN SURESTE: CASO TABASCO.

Café Chiapas

Café Veracruz

Charanda

Mango Ataulfo del

Soconusco de Chiapas

Mezcal

Olinalá

Sotol

Talavera
Café verde o 12 regiones de Chiapas: Ocozocoautla, San tostado molido, de Cristobal de las Casas, Copainalá, Comitán, la especie Cooffea Ángel Albino Corzo, Pichucalco, Bochil, arabica, Palenque, Ocosingo, Yajalón, Motozintla y perteneciente a la Tapachula. En las que se engloban un total familia de las de 82 municipios.

rubiáceas.

Café verde o Todo el Estado de Veracruz, siempre que se tostado. siembre a más de 750 metros de altitud.

Bebida alcohólica 16 municipios de Michoacán: Tangancícuaro, resultado de la Cotija, Tocumbo, Los Reyes, Peribán, fermentación y Uruapan, Nuevo Parangaricutiro, Tacítaro, destilación del jugo Taretán, Ziracuaretiro, Salvador Escalante, de la caña de Tacámbaro, Nuevo Urecho, Gabriel Zamora, azúcar. Ario y Turicato.

Chile habanero Yucatán, Campeche y Quintana Roo.

fresco, en estado inmaduro o maduro, encurtido, en pasta, deshidratado, entero, en polvo y en salsas.

Mango Ataulfo

13 municipios de Chiapas: Acacoyagua, Acapecahua, Escuintla, Tuzantán, Huixtla, Villa Comaltitlán, Mazatán Huehuetán, Tapachula, Tuxtla Chico, Metapa, Frontera Hidalgo y Suchiate.

Bebida alcohólica Durango, Zacatecas, San Luis Potosí, destilada de Guerrero, Tamaulipas (11 municipios), maguey. Guanajuato (2 municipios), Michoacán (29 municipios), Puebla (115 municipios) y Oaxaca (8 municipios).

Artesanía de Municipio de Olinalá, Guerrero madera que se manufactura con materia prima del municipio de

Olinalá, Guerrero.

Bebida alcohólica Chihuahua (67 municipios), Coahuila (38 destilada de municipios) y Durango (38 municipios).

maguey.

Loza artesanal de Puebla (Cholula, Puebla, Tecali y Atlixco) y Talavera.
Tlaxcala (San Pablo del Monte) 
LA INFLUENCIA DE LAS DENOMINACIONES DE ORIGEN EN EL DESARROLLO ECONÓMICO DE LA REGIÓN SURESTE: CASO TABASCO.

$\begin{array}{lll}\text { Tequila } & \begin{array}{l}\text { Bebida alcohólica } \\ \text { destilada de agave } \\ \text { llamada tequila. }\end{array} & \begin{array}{l}\text { Tamaulipas } \\ \text { municipios), Jalisco }(125 \text { municipios), Nayarit (8 } \\ \text { Michoacán (29 municipios) y Guanajuato (7 } \\ \text { municipios). }\end{array} \\ \text { Vainilla de Papantla } & \begin{array}{l}\text { Extracto y derivados } \\ \text { del fruto maduro }\end{array} & \text { Puebla (19 municipios) y Veracruz (20 } \\ \text { beneficiado de la } \\ \text { orquídea vanilla } \\ \text { planifolia Andrews. }\end{array}$

Fuente: Elaboración propia en base a la información obtenida del documento DO Orgullo de México editado por la SE y el IMPI, 2016, así como el extracto de la DO Cacao Grijalva publicado el 29 de agosto de 2016.

\section{El cacao en el Estado de Tabasco}

Los principales países que cultivan Cacao son África del Oeste, América Central, Sudamérica y Asía. Los ocho mayores países productores cacao del mundo son Costa de Marfil, Ghana, Indonesia, Nigeria, Brasil, Camerún, Ecuador y Malasia, estos países representan el 90\% de la producción mundial. México, a pesar de sus condiciones climáticas para ser uno de los principales productores, ocupa el onceavo lugar de producción, participando con menos del $2 \%$ de la producción mundial (Cacaoméxico.org, 2016).

El cacao es un producto producido principalmente por el estado de Tabasco, el cual produce $66 \%$ de la producción nacional, seguido de Chiapas que produce el $33 \%$, el resto lo produce Oaxaca, Guerrero y Veracruz (Cacaoméxico.org, 2016).

Este producto se cultiva desde la época prehispánica, sin embargo, es a partir de los años treinta cuando comienza a tomar importancia como un producto explotable para la región, sobre todo en la gestión del Gobernador Garrido Canabal. Los productores de cacao lo consideraban un cultivo alternativo, ya que sus ingresos no solo dependían de esta actividad, sino que estos además eran arroceros, cultivaban caña de azúcar, se dedicaban a la ganadería, eran jornaleros.

En Tabasco el cacao es un cultivo con una larga tradición. En 1995 la región productora del estado de Tabasco se encontraba distribuida en diez de los diecisiete municipios: Huimanguillo, Paraíso, Cárdenas, Tacotalpa, Balancán, Nacajuca, Comalcalco, Cunduacán, Jalpa de Méndez y Teapa.

De 2001 a 2009 la producción de cacao disminuyó 47\%, pasando de 46,700 a 24,700 toneladas, vinculada esta caída principalmente al moniliasis, así como el envejecimiento de las plantaciones y descuido de los cultivos. 

LA REGIÓN SURESTE: CASO TABASCO.

En Tabasco se cultiva el cacao forastero mayormente, por la resistencia a las enfermedades y por su mayor rendimiento.

El Gobernador del Estado de Tabasco, el Lic. Arturo Núñez, señala que el cacao es un producto esencial para el desarrollo del estado. Desde el punto de vista económico contribuye a la generación de miles de empleos; en material ambiental permite la conservación de un ecosistema único, y desde la perspectiva histórica, nos da identidad por ser un producto totalmente originario de Tabasco.

\section{Regionalización del Estado de Tabasco}

La regionalización a que se hace mención por parte de la Secretaría de Desarrollo Agropecuario, Forestal y Pesquero (SEDAFOP), delegación Tabasco, surge a partir del 29 de junio de 1994, fecha en la cual el Estado de Tabasco fue regionalizado, basado en el Programa Estatal de Desarrollo Urbano y Ordenamiento Territorial, en donde se adecua la cartografía regional publicada en el periódico oficial número 5,406. Esta regionalización divide al Estado de Tabasco en dos regiones: la región Grijalva y la región Usumacinta, estas regiones a su vez se dividen en cinco subregiones; dentro de estas subregiones se agrupan los municipios, los cuales comparten determinadas características (INAFED, 1999). Esta división es utilizada por CONAGUA, ya que se basa en los dos grandes ríos que dan vida al Estado.

La región Grijalva, lleva el nombre del río Grijalva, representa el $48.94 \%$ del territorio tabasqueño. Se conforma por tres subregiones Chontalpa, Centro y Sierra, los municipios que integran estas subregiones son: Huimanguillo, Cárdenas, Comalcalco, Cunduacán, Paraíso, Jalpa de Méndez, Nacajuca, Centro, Jalapa, Teapa y Tacotalpa.

La región Usumacinta, recibe el nombre del rio que riega los municipios de Centla, Jonuta, Emiliano Zapata, Balancán y Tenosique, el municipio de Macuspana también pertenece a esta región. Sus subregiones son: Pantanos y Ríos.

\section{Caso Denominación de Origen Chile Habanero de la Península de Yucatán}

En el caso de la DO Chile habanero de la Península de Yucatán, en un inició los productores de chile habanero del Estado de Yucatán A. C. solicitaron la marca colectiva Chile Habanero de Yucatán, el 31 de julio de 2003 al IMPI bajo el número de expediente 612526, esta marca colectiva fue otorgada el 25 de noviembre de 2003, donde el titular era asociación civil Chile habanero de Yucatán, con número de registro 814176, ésta marca colectiva fue solicitada para proteger productos contemplados en la clase 31 correspondiente a Chile habanero (IMPI, 2003). 

LA REGIÓN SURESTE: CASO TABASCO.

El Presidente de Chile Habanero de Yucatán A.C., el C. Juan Carlos Ledón Vadillo, solicita al IMPI la Declaración de Protección de la DO "CHILE HABANERO DE YUCATÁN" el 23 de marzo de 2005, dando inicio al trámite el 2 de agosto de 2006, se publica el 17 de octubre de 2007 en el Diario Oficial de la Federación un extracto de esta solicitud, a fin de que objeciones, y aportara las pruebas que estimara pertinentes. Se reciben tres escritos, el primero de fecha 15 de noviembre de 2007 de la Secretaria de Desarrollo Rural y Fomento Industrial y Comercial del Estado de Campeche, en el cual solicita la adhesión del Estado de Campeche a la solicitud de la DO en cuestión; el segundo escrito de fecha 20 de noviembre del mismo año de la Secretaria de Desarrollo Agropecuario Rural e Indígena, y de Desarrollo Económico de Quintana Roo, en el cual solicita de igual forma la adhesión del Estado de Quintana Roo a dicha solicitud de DO antes señalada, por último el escrito de fecha 29 de octubre del mismo año de los gobernadores del Estado de Yucatán, Campeche y Quintana Roo, en el cual solicitan de común acuerdo, se efectúe el cambio del nombre de la DO a "CHILE HABANERO DE LA PENÍNSULA DE YUCATÁN", escritos que determinaron improcedente. Ante esta situación el IMPI, otorga el 3 de octubre de 2008 otorga la protección a DO "CHILE HABANERO DE YUCATÁN" (DOF, 2008).

Posterior a esta declaratoria, el 8 de septiembre de 2009 Ivonne Aracelly Ortega Pacheco, Gobernadora del Estado de Yucatán, Juan Carlos Ledón, Presidente de la Asociación Civil Chile habanero de Yucatán A. C, Félix Arturo González Canto, Gobernador del Estado de Quintana Roo, Jorge Manuel del Toro Chávez, productor y Director General de Hidroponía Maya S. A. de C. V., Jorge Carlos Hurtado Valdez, Gobernador del Estado de Campeche y Alberto Córdova Gaspar, Presidente del Comité Sistema Producto Chile de Campeche A. C. Presentan la solicitud de Declaración de Protección de la DO "CHILE HABANERO DE LA PENÍNSULA DE YUCATÁN" (Declaratorio General de Protección de la DO Chile Habanero de la Península de Yucatán, 2010).

El 31 de mayo de 2010 el IMPI otorga la protección a la DO "CHILE HABANERO DE LA PENINSULA DE YUCATÁN", el titular de la DO es el Estado Mexicano, y ésta DO solo podrá ser usada mediante la autorización que expida el IMPI, a las personas físicas o morales que reúnan los requisitos establecidos en la Ley de la Propiedad Industrial.

El mismo año en el que fue otorgada la DO, cuatro meses antes, el 30 de junio de 2008, fue solicitada la cancelación de la marca colectiva y se tomó nota de la cancelación por parte del Instituto el 4 de septiembre de 2008 (IMPI, 2008). 

LA REGIÓN SURESTE: CASO TABASCO.

En esta solicitud de DO, se encuentran involucrados dos actores esenciales, en un primer plano los productores del chile habanero, y en el segundo los Gobiernos de Yucatán, Campeche y Quintana Roo. Existe una participación activa por parte de las Asociaciones, tal como se desprende de los documentos consultados.

Esta participación se ve reflejada en todos los sectores, incluso existe investigación + desarrollo involucrado que respalda a este producto, un ejemplo de esto es la Industria Agrícola Maya S. A. de C. V., la cual fue creada en el año 2002, con el objeto social de producir, transformar y comercializar productos del campo, así como la de realizar actividades de investigación y desarrollo para obtener procesos y productos innovadores con alto valor agregado, que le permiten innovar y mejorar los productos que se ofrecen actualmente (INDAMAYA, 2016).

\section{Caso Denominación de Origen Cacao Grijalva}

En el caso del Estado de Tabasco, los productores de cacao del municipio de Comalcalco, Tabasco, la Sociedad denominada Grupo de Productores Agrícolas de Innovación Tecnológica en Cacao Comalcalco S. P. R. de R. L. a través de su Representante el C. Rodolfo Arias Naranjo, el 13 de noviembre de 2013 realizan la solicitud de la marca colectiva TROPICAO, para respaldar productos contemplados en la clase 30 correspondientes a CACAO y CHOCOLATE, con el número de expediente 629335, dicha marca colectiva fue otorgada el 7 de abril de 2004 con el registro número 828668 (IMPI, 2003). Esta marca caducó el 24 de octubre de 2013, pasando inadvertida.

El 26 de agosto de 2013, la SEDAFOP del Estado de Tabasco solicitó la Declaración General de Protección de la DO "CACAO GRIJALVA". Esta Secretaría a través de su representante, expresa su interés jurídico, entre otros, en los artículos 12, 26 fracción X y 36 de la Ley Orgánica del Poder Ejecutivo del Estado de Tabasco; 5 fracción I, 51, 52 fracciones I, VII y VIII de la Ley Agrícola para el Estado de Tabasco en correlación con el Plan Nacional de Desarrollo; de donde se desprende que dicha Secretaría fue creada para coordinar lo conducente para el mejor desarrollo de las actividades agropecuarias, forestales y de pesca, así como promover la participación de los municipios, de las organizaciones sociales y de productores, en la planeación, elaboración de programas y acciones para el desarrollo y fomento de la actividad agrícola, procurando el aprovechamiento integral y sustentable de los recursos naturales de la entidad (DOF, 2016). Es interesante el hecho de que esta solicitud la realiza dicha Secretaría sin el apoyo o respaldo de organización de productores contrario a lo que sucede al proceso de la DO del Chile Habanero de la Península de Yucatán, esta Secretaria tabasqueña únicamente hace alusión a las facultades 

LA REGIÓN SURESTE: CASO TABASCO.

jurídicas otorgadas y con las cuales sustenta su interés jurídico para llevar a cabo esta solicitud.

Esta DO ampara el cacao verde o tostado/molido de la especie Theobroma Cacao, con las características, componentes, forma de extracción y proceso de producción, elaboración y sus modos de empaque, embalaje o embasamiento.

Dentro del documento la SEDAFOP, delegación Tabasco señala que entre las variedades de cacao se encuentran: el criollo, el trinitario y el forastero. La población de cacao cultivada en la región Grijalva de Tabasco, en su mayoría, pertenecen al grupo genético Trinitario, producto de la hibridación natural entre los criollos nativos y los forasteros introducidos a principios de siglo. Predominan las formas de fruto amelonado, calabacillo, angoleta y cundeamor.

Los lugares de extracción, producción o elaboración del producto que se trata proteger con esta DO es la Región Grijalva de Tabasco, integrada por tres subregiones productivas, denominadas Chontalpa, Sierra y Centro, subregiones donde se produce la totalidad del "Cacao Grijalva" del Estado (Declaración General de Protección de la DO "Cacao Grijalva", 2016).

El 16 de agosto de 2016 el IMPI otorga la protección a la DO "Cacao Grijalva", al igual que las otras 14 DO, el titular es el Estado Mexicano, y de igual forma solo podrá ser usada mediante la autorización que expida el IMPI, a las personas físicas o morales que reúnan los requisitos establecidos en la Ley de la Propiedad Industrial.

\section{Planteamiento del problema}

A pesar de tratarse de una certificación relevante para un producto del Estado de Tabasco los pequeños y medianos productores de cacao, así como los consumidores del mismo producto perteneciente a las subregiones centro y sierra del Estado de Tabasco, no tiene conocimiento respecto del otorgamiento de la DO "CACAO GRIJALVA" otorgado en fechas recientes por el IMPI al Estado de Tabasco, más aún desconocen las razones por las cuales se le dio este nombre al cacao producido en Tabasco, ya que el nombre hace alusión a otros factores que no se relacionan con el cacao. El territorio en el cual se produce el cacao en años anteriores contemplaba 10 de los 17 municipios del Estado de Tabasco, llama la atención que en la denominación de origen se agrega el municipio del Centro, el cual no contaba con presencia como principal productor, en años en los que el cacao estuvo en su esplendor en Tabasco. 

LA REGIÓN SURESTE: CASO TABASCO.

Esto nos lleva a realizarnos la siguiente pregunta ¿El otorgamiento de la DO "Cacao Grijalva" representa realmente una influencia favorable en la economía de la región? ¿Esta DO cumple con la función identificadora del producto en cuestión? Para responder estos cuestionamientos, el presente estudio se desarrolló con productores independientes de la subregión Centro de la Región Grijalva, tal como lo señala la Declaración de DO del Cacao Grijalva.

\section{MATERIAL Y MÉTODO}

El presente estudio tiene un enfoque cualitativo de corte transversal, no experimental. Los métodos utilizados fueron documental, observación directa y método hipotético-deductivo, de igual forma se llevó a cabo un análisis comparativo entre ambas denominaciones "Cacao Grijalva" y "Chile Habanero de la Península de Yucatán".

Presenta dos etapas, en la primera se realiza la parte documental, a fin de contar con el marco correspondiente para realizar la documental y la aplicación del método hipotéticodeductivo y dar paso a la segunda etapa.

En la segunda etapa se realiza la parte cualitativa, utilizando como técnica el cuestionario cerrado, consisten en la aplicación de esta herramienta a los productores independientes de cacao de la subregión Centro de la Región Grijalva, para lo cual se realizó una muestra de la región ubicada en la Ranchería Rio Viejo, Ixtacomitán, Boquerón 1ra, 2da y 3ra sección, donde se presenta mayor producción ya que se encuentra cerca de la región chontalpa, de la muestra se obtuvo el resultado de 250 productores, se eligió esta región en virtud de que en la Declaración de DO del Cacao Grijalva, se señala al municipio de Centro dentro de los once municipios productores del Cacao Grijalva, se aplicó dicho cuestionario a fin de determinar el impacto e influencia de la DO en el sector productivo del municipio.

\section{Población}

Los sujetos de estudio en esta investigación son 250 productores independientes de cacao de la subregión Centro de la Región Grijalva, muestra elegida de manera aleatoria.

\section{Instrumentos y procedimientos}

Para esta investigación, en la primera etapa se realizó el análisis hipotético-deductivo, tomando la información obtenida en la primera etapa con los resultados de la investigación documental, en base a esto, se elaboró el instrumento correspondiente, mismo que se aplicó a los 250 productores de cacao de la región. El instrumento, la observación directa y la 

LA REGIÓN SURESTE: CASO TABASCO.

documentación obtenida se basó respecto de la DO, su funcionamiento, su aplicación en el territorio nacional, regional y estatal, así como de la productividad cacaotera y su importancia en el Estado de Tabasco, a fin de determinar el impacto de la declaración de DO Cacao Grijalva en la economía regional, para lo cual se realizó un análisis de información cruzada obtenida de las legislaciones nacionales e internacionales correspondientes, las publicaciones de propiedad intelectual e industrial, las bases de datos del IMPI, las declaraciones de las denominaciones de origen en el diario oficial de la federación, así como diarios, noticias, entre otros.

En la segunda etapa, se aplicó un cuestionario con 15 preguntas cerradas. Dichas preguntas fueron aplicadas por las investigadoras de este proyecto, cada entrevista duró entre 10 y 15 minutos.

\section{RESULTADOS}

Del resultado derivado de la aplicación del cuestionario cerrado, las tres preguntas corresponden al estudio sociodemográfico, la primera referente a la edad, la segunda al último grado escolar; y por último, la ocupación principal de estos productores. Las doce preguntas restantes se relacionan directamente con el tema de la Denominación de origen.

Los productores de cacao en su mayoría son productores que se encuentran entre los 46 y 50 años, seguidos de los productores de 50 años en adelante con 55 productores y por último 35 productores entre 36 a 45 años. Del análisis de estos datos, se identifica que la mayoría de la población pasa de los treinta años, y que los menores de 35 años actualmente se encuentran trabajando en otros lugares o se encuentran estudiando, no existe la continuidad de la actividad económica.

Respecto a la segunda pregunta, referente al último grado escolar, aportó los siguientes datos 25 productores no tienen ningún tipo de estudio por lo tanto no saben leer pero sí saben contar, 155 cuenta con primaria, 65 tienen secundaria, 10 tienen preparatoria, 14 carrera técnica y un productor cuenta con carrera universitaria. Estos datos nos reflejan que existe un nivel académico básico en la mayoría de los productores lo que no permite un crecimiento económico relevante.

En la tercera pregunta en relación a la ocupación 180 son empleados, 20 empresarios, 0 estudiantes, 10 amas de casa y 40 se dedican a otra ocupación. Todos ellos tienen de manera alternativa el cultivo del cacao, ya que sus ingresos no dependen de esta actividad. 

LA REGIÓN SURESTE: CASO TABASCO.

Esta información muestra que ninguno de los productores toma la como actividad principal el cultivo del cacao.

Algunos argumentaron que esto se debe en primera instancia que ya no se cuentan con la cantidad de hectáreas cultivadas del que había hace 25 años, esto se debe en gran parte a la plaga que invadió las matas de cacao, luego al hecho que la cosecha no resultaba redituable, puesto que se invertía más en trabajadores para que sacaran el cacao que en lo que se obtenía de la venta.

En la pregunta 4, respecto si sabían que era una DO, 236 productores desconocían dicha figura, 14 han escuchado o visto un comercial al respecto, sin embargo, esto nos permitió conocer que a pesar de la promoción que ha hecho la secretaría estatal no ha logrado permear a los pequeños productores de cacao. Los que habían visto o escuchado no sabían en qué consistía la DO.

La pregunta anterior, se encuentra ligada a la pregunta 5, ya que se cuestiona si tiene conocimiento que el IMPI otorgó una DO al Estado de Tabasco, 236 no tenían conocimiento, 14 habían escuchado al respecto por el radio.

En la pregunta 6 , se les cuestionó si conocían a que producto se le otorgó la DO, 10 contestaron afirmativamente y 240 lo desconocían; sin embargo, en la pregunta 7 en la cual se les aplicó únicamente a los 10 productores que contestaron afirmativamente, en esta pregunta se daba a elegir un producto entre tilapia, cacao, chile amashito, mango o robalo y 7 contestaron erróneamente el producto y 3 acertaron con el cacao. La pregunta 8, 9, 10 y 11 se encuentran relacionadas ya que fueron aplicadas únicamente a los 10 productores que contestaron afirmativamente la pregunta 8 . En la pregunta 8 se cuestiona si tienen conocimiento de que DO se le dio al cacao, 10 contestaron que afirmativamente y 240 desconocían el nombre que se le dio a dicho producto. Sin embargo, en la siguiente pregunta, sólo fue contestada por los 10 productores que contestaron afirmativamente la pregunta anterior y quedó claro que realmente 2 de éstos identifican la DO como "Cacao Grijalva", los demás señalaron Tilapia Grijalva, Cacao Tabasco, Mango Tabasco y Robalo Grijalva, en la pregunta 10 se les pregunta si tienen conocimiento de la razón por la cual el Gobierno del Estado de Tabasco decidió asignarle ese nombre, 2 respondieron afirmativamente y 8 lo desconocían. Los 2 productores que respondieron afirmativamente señalaron en la pregunta 11, que la razón fue por incluir a Chiapas. 

LA REGIÓN SURESTE: CASO TABASCO.

En la pregunta 12, respecto de los municipios que forman parte de la DO, todos señalaron que los 17 municipios del Estado de Tabasco, lo cual es incorrecto, ya que únicamente 11 municipios del Estado de Tabasco están contemplados dentro de la declaración de la DO de Cacao Grijalva.

Las preguntas 13 y 14 se encuentran ligadas, ya que en la primera se le cuestiona al productor si tiene conocimiento del tipo de producción de cacao llevará la DO, 246 lo desconocían y 4 contestaron afirmativamente, y en la siguiente pregunta concretaron a 3 criollo y 1 trinitario.

En la última pregunta, se le pregunta a los productores quien es el titular de los derechos de la DO, 240 señalaron que lo desconocían, 5 señalaron que los productores, 3 la SEDAFOP y 2 el Gobierno de Tabasco.

\section{CONCLUSIONES}

Muestran que la DO, en el caso de la Península de Yucatán por el proceso que se llevó a cabo, se encuentran involucrados los productores de los tres estados, así como la inversión en investigación, desarrollo, innovación, comercialización, capacitación e interés por parte de todos los sectores, permite que tenga una aceptación mucho más completa con los consumidores y la economía de la región. Sin embargo, esto no se ve reflejado en el caso de la DO "Cacao Grijalva", ya que esta denominación tiene un proceso en el cual únicamente se encuentra promoviéndolo la SEDAFOP, sin el consenso y conocimiento por parte de los productores pertenecientes a municipios que no pertenecen al grupo de la subregión de la Chontalpa. Tabasco a pesar de contar con las condiciones necesarias para desarrollar el cacao que se protege con la DO, requiere de mayor esfuerzo reflejado en capacitación del capital humano, interés e involucramiento, aunado a la inversión económica, ya que como es cierto, desde el 2001 a 2009 se produjo una pérdida considerable de estos cultivos que las condiciones económicas y ambientales no han permitido la recuperación del sector.

Es importante contar con el respaldo de la población como consumidores, productores, comerciantes e investigadores para obtener las metas planteadas, de lo contrario la obtención de esta denominación no servirá de nada.

En relación a la respuesta a la pregunta principal de este estudio ¿El otorgamiento de la DO "Cacao Grijalva" representa realmente una influencia favorable en la economía de la región? 
LA INFLUENCIA DE LAS DENOMINACIONES DE ORIGEN EN EL DESARROLLO ECONÓMICO DE LA REGIÓN SURESTE: CASO TABASCO.

¿Esta DO cumple con la función identificadora del producto en cuestión? De acuerdo a los resultados obtenidos, se responde a la primera pregunta, que se tiene un arduo trabajo por delante para lograr colocar la DO en los estándares establecidos por las otras 14 DO nacionales, por otro lado, en base a los mismo datos obtenidos, puede advertirse que realmente no cumple la función de IG, debido a que existe una figura de propiedad industrial distinta; es decir, al producto se le otorgó una marca, ya que el nombre no corresponde a indicar a qué lugar pertenece el cacao, incluso sería apropiado señalar que la denominación que necesitaba este producto es Cacao Tabasco o incluso Cacao de la Chontalpa. 
LA INFLUENCIA DE LAS DENOMINACIONES DE ORIGEN EN EL DESARROLLO ECONÓMICO DE LA REGIÓN SURESTE: CASO TABASCO.

\section{REFERENCIAS BIBLIOGRÁFICAS}

Cacaoméxico.org, principales países productores de Cacao, Recuperado de: http://www.cacaomexico.org/?page_id=201

Declaratorio General de Protección de la Denominación de Origen Chile Habanero de Yucatán, publicada en el Diario Oficial de la Federación el 10 de octubre de 2008.

Declaratoria General de Protección de la Denominación de Origen Chile Habanero de la Península de Yucatán, publicada en el Diario Oficial el 4 de junio de 2010.

Declaración General de Protección de la Denominación de Origen "Cacao Grijalva", publicada en el Diario Oficial de la Federación el 29 de agosto de 2016.

Instituto Mexicano de la Propiedad Industrial (2008). Oficio con número de folio 20080453620.

INAFED, Enciclopedia de los municipios y delegaciones de México, Tabasco, 1999.http://www.inafed.gob.mx/work/enciclopedia/EMM27tabasco/regionalizacion .html.

Instituto Mexicano de la Propiedad Industrial (2016). DO Orgullo de México. México: Pax.

Instituto Mexicano de la Propiedad Industrial (2003a). Servicio de consulta externa sobre información de marcas, expediente 612526 respecto de la marca mixta Chile habanero de Yucatán.

Instituto Mexicano de la Propiedad Industrial (2003b). Servicio de consulta externa sobre información de marcas, expediente 629335 respecto de la marca colectiva mixta Tropicao y diseño.

Jatib, M. I., (1996) Propuesta de implementación del sistema de denominaciones de origen en Argentina, exposición en el I Seminario de Denominaciones de Origen, Buenos Aires.

Ley de la Propiedad Industrial (2016). Publicada en el Diario Oficial de la Federación el 27 de junio de 1991, última reforma publicada el 1 de junio de 2016. 
LA INFLUENCIA DE LAS DENOMINACIONES DE ORIGEN EN EL DESARROLLO ECONÓMICO DE LA REGIÓN SURESTE: CASO TABASCO.

Organización Mundial de la Propiedad Intelectual (2016a) Indicaciones geográficas ¿Qué es una indicación geográfica? Ginebra, Suiza: Autor, p. 25.

Organización Mundial de la Propiedad Intelectual (2016b). El Sistema de Lisboa, protección internacional de identificadores de productos típicos de zonas geográficas específicas. Ginebra, Suiza: Autor.

Ortíz, M. A. (Coord.), Ley de la Propiedad Industrial, comentada por la Asociación Mexicana para la Protección de la Propiedad Intelectual AMPPI. México: Porrúa. 2015, pp. 322, 325 у 333.

Tabasco y el cacao: Crónica de una tradición. Claridades agropecuarias, 16. México, pp. 2-23. 